\title{
E-Field Distribution modeling in a Homogeneous Phantom for a rapid SAR measurement
}

\author{
Olivier Merckel and Jean-Charles Bolomey \\ Supélec/DRE \\ 3 rue Joliot-Curie \\ Gif-sur-Yvette, FRANCE \\ e-mail: Olivier.Merckel@,supelec.fr
}

\author{
Gilles Fleury \\ Supélec/Me \\ 3 rue Joliot-Curie \\ Gif-sur-Yvette, FRANCE \\ e-mail: Gilles.Fleury@supelec.fr
}

\begin{abstract}
Specific Absorption Rate (SAR) designates the electromagnetic power density deposited per unit mass of biological tissue. SAR measurements are required to assess the compliance of mobile phones with existing standards and recommendations. The use of homogeneous phantoms leads to a relatively simple distribution of the electric field. Several ways of determining the decrease of the electric field in function of depth are here explored. The choice of a pseudo propagation constant allows to drastically reduce the number of E-field measurement points needed for the SAR calculation. The measurement time is then reduced to less than a minute, while the standard way takes about 10 minutes for a complete measurement of almost thousand E-field data points.
\end{abstract}

Keywords: SAR, fast, propagation, parametric, ellipsoid.

\section{Introduction}

The experimental electromagnetic dosimetry of mobile phones has much developed since the beginning. Most of the existing dosimetric facilities utilize automatic positioning systems to move an E-Field measuring probe, with the help of robotized arms [1], or three axes displacement systems in order to achieve SAR (Specific Absorption Rate) measurements. The European Standard prEN50361 [2] details the ways to measure the SAR in a head-like phantom, and the maximum value of the SAR averaged in $10 \mathrm{~g}$ allowed. The electromagnetic properties of the liquid filling the phantom are similar to those of the brain. According to the new European standard, a complete phone test lasts about half a day, pointing out a new concern: the rapidity of SAR measurement. A rapid (less than 1 minute) and non-invasive SAR measurement solution, based on a probe integrated in a spherical phantom around which the phone under test turns, has been still developed [3]. The rapid SAR measurement method that we propose here is fully compatible with popular instrumentation and, hence, can be directly implemented on most existing SAR measurement facilities using mechanical scanning of an E-field probe [4]. The number of electric field data is reduced by a factor 30 that allows dividing the measurement time by a factor 10 approximately.

The physical quantity that we measure, the maximum SAR (1) averaged in $1 \mathrm{~g}$ and $10 \mathrm{~g}$ of contiguous tissues, is the volumetric integration of the quadratic electric field, weighted by the conductivity $\sigma$ and the density $\rho$ of the media:

$$
S A R=\frac{\sigma\|E\|^{2}}{\rho}
$$

with $\|E\|(\mathrm{V} / \mathrm{m})$ the E-field norm, $\sigma(\mathrm{S} / \mathrm{m})$ the conductivity of the medium, and $\rho\left(\mathrm{kg} / \mathrm{m}^{3}\right)$ its density.

The simplicity of the E-field distribution in the phantom, the more often in a long spot perpendicularly to the direction of propagation, its reproducibility between the different mobile phones, allows to consider generic parametric models of the E-field able to fit the principal electromagnetic characteristics of the phones. The number of parameters of those models (between 5 and 11) is sufficiently low to reconstruct the electric field in the whole data volume, from a small number of data, in a very reasonable computation time (on the order of one second). The acquisition time is then drastically reduced. The results presented in the last section show SAR reconstructions of commercial phones from three models involving a strict ellipsoidal approach, and two hybrid ellipsoidal-plane wave or mean propagation constant approach, those one trying to reduce the number of data points needed to a few ones distributed in a plane. These approaches are based on a long experience in SAR measurements, and in parametric methods applied to physical measurements. Their development has been possible with the help of the hundreds SAR tests of commercial phones, and are a result of the research effort conducted to improve the accuracy and the rapidity of the Supélec dosimetric facility.

\section{Physical characteristics of the E-field}

\section{Decrease of the E-Field in function of depth}

The construction of the mathematical models representing the electric field is issued from the conclusions of systematic SAR tests on a hundred of real mobile phones (at 2 frequencies - 900 $\mathrm{MHz}$ and $1800 \mathrm{MHz}$ ).

The analysis of the E-field in function of depth in the head-like phantom shows an exponential decay (see Fig. 1) for more than 90 $\%$ of the tested phones. The curves corresponding to each tested phone are distributed around the line corresponding to the theoretical propagation constant $\alpha$ of the plane wave (2).

$$
E(x, y, z)=E(x, y, d) e^{-\alpha(z-d)}
$$

For the standard materials used, and for a plane wave, $\alpha=-29.4$ $\mathrm{Np} / \mathrm{m}$ at $900 \mathrm{MHz}$, and $\alpha=41.4 \mathrm{~Np} / \mathrm{m}$ at $1800 \mathrm{MHz}$.

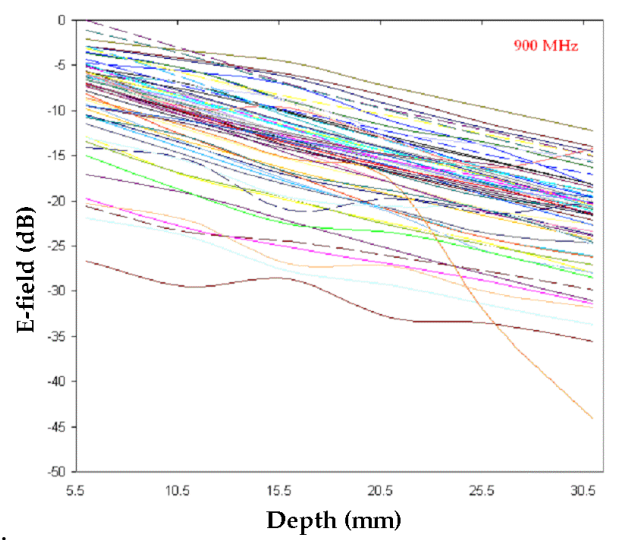

Figure 1. Depth decrease of the maximum normalized E-Field, for 64 phones at $900 \mathrm{MHz}$. 
The $10 \%$ of mobile phones a bit far away from that observation concerns the phones for which measurement is too noisy to be significant. In those cases, the E-field level is at the limit of sensibility of the probe $(\approx 1 \mathrm{~V} / \mathrm{m})$, and is almost drowned in the noise

\section{Attenuation and propagation}

In a homogeneous and isotropic media (inside the phantom), the Efield can be represented as a plane wave sum. When the media is conductive, it is however necessary to take into account the introduction of the losses. Furthermore, the presence of evanescent waves has to be studied. The E-Field inside the phantom can be seen as the integration of the angular plane wave spectrum $\mathbf{A}(\mathbf{u})$ in the considered domain $\boldsymbol{\Omega}$ :

$$
\mathbf{E}(\mathbf{r})=\iint_{\mathbf{\Omega}} \mathbf{A}(\mathbf{u}) \exp (-j k \mathbf{r} . \mathbf{u}) d \mathbf{\Omega}(\mathbf{u})
$$

with $\mathbf{u}=\{\alpha, \beta, \chi\}, \alpha^{2}+\beta^{2}+\chi^{2}=1$, and $\gamma= \pm \sqrt{1-\left(\alpha^{2}+\beta^{2}\right)}$.

Each plane wave can be represented with the following standard form, in which the term $k$, the propagation constant, is a unique complex scalar (4).

$$
\mathbf{E}(\mathbf{r})=\mathbf{E}_{0}(\mathbf{r}) \exp (-j k z)
$$

In function of the physical characteristics of the media $k^{2}=\omega^{2} \mu_{0} \varepsilon_{0} \varepsilon_{r}$, with $\quad \varepsilon_{r}=\varepsilon_{r}^{\prime}-j \varepsilon_{r}^{\prime \prime}$, the complex propagation "constant" $k$ is expressed as follows,

$$
k=\frac{\omega}{c}\left(\sqrt{\frac{\left|\varepsilon_{r}\right|+\varepsilon_{r}^{\prime}}{2}}-j \sqrt{\frac{\left|\varepsilon_{r}\right|-\varepsilon_{r}^{\prime}}{2}}\right)
$$

with $k=k^{\prime}-j k^{\prime \prime}$. The plane wave is represented in the homogeneous conductive media with the equation (6) :

$$
\mathbf{E}(\mathbf{r})=\mathbf{E}_{0}(\mathbf{r}) \exp \left(-j k^{\prime} z\right) \exp \left(-k^{\prime \prime} z\right)
$$

The imaginary part of $k$ is responsible for the attenuation $\alpha$ of the wave, due to the losses in the equivalent liquid. We then introduce $\alpha=\operatorname{Im}(k)$, expressed in Np. $\mathrm{m}^{-1} . k^{\prime \prime}$ represents somewhat the skin thickness in the media. The real part $k^{\prime}$ of the wave vector allows defining the wavelength in this media.

For a standard phantom and the plane wave, the values of $\alpha$ regarding the frequency are:

Table 1. plane wave attenuation in a biological media

\begin{tabular}{|c|c|c|}
\hline Plane wave & $900 \mathrm{MHz}$ & $1800 \mathrm{MHz}$ \\
\hline$\alpha(\mathrm{Np} / \mathrm{m})$ & $-29,4$ & $-41,4$ \\
\hline
\end{tabular}

The electric field inside the phantom is not a plane wave but a linear combination of plane waves. In order to describe the distribution of the E-field in a phantom with simple models and to limit the number of parameters, it is necessary to find a convenient way of determination for a unique propagation constant $\alpha$. The calculation of $\alpha$ from a few points distributed in function of depth on a line perpendicular to the planar phantom interface doesn't allow describing the exact behaviour of the electric field, but gives an interesting approach. This principle has still been applied to describe the decrease of the electric field in a phantom for a rapid SAR measurement [3].

The value of $\alpha$ is calculated for each phone, at first time as the slope value of the lines observed in Fig. 1. The Fig. 2 shows the values of $\alpha$ obtained from the maximum amplitude of the electric field, and from the averaging of all the propagation constants observed in the measurement space. The horizontal line represents the corresponding plane wave propagation constant value, at $900 \mathrm{MHz}$ and $1800 \mathrm{MHz}$. The measurement is much less perturbed at $900 \mathrm{MHz}$ than at $1800 \mathrm{MHz}$, where the attenuation is stronger. The sensitivity of the electric field measuring probe (about $1 \mathrm{~V} / \mathrm{m}$ ) is then, for some phones, too low to give a good accuracy for the measurement.

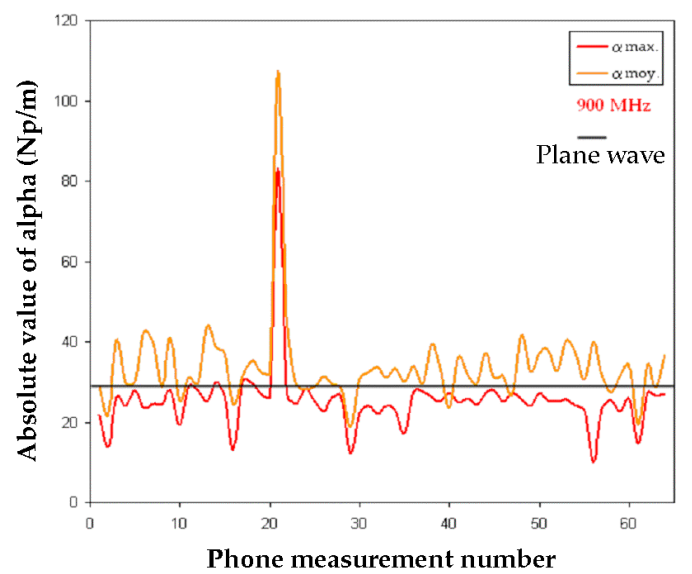

Figure 2. Absolute values of the mean and maximum propagation "constant" $\alpha\left(\mathrm{Np} . \mathrm{m}^{-1}\right)$ at $900 \mathrm{MHz}$ for 64 phones

\section{Histograms of the parameter $\alpha$}

We have represented the distribution of the propagation constant in function of its values, by means of the histograms of the Fig. 3. With the corresponding distribution functions, we then deduced the $10 \%$ and $90 \%$ quantiles. The $q$ order quantile is the inverse function for the distribution function. It gives the value $\alpha_{\mathrm{n}}$ so that $\operatorname{Prob}\left(\begin{array}{ll}\alpha & \alpha_{\mathrm{n}}\end{array}\right)=q$. It allows obtaining an estimation of the upper bound of $\alpha$ with a probability $q$ that this value will not be underestimated.
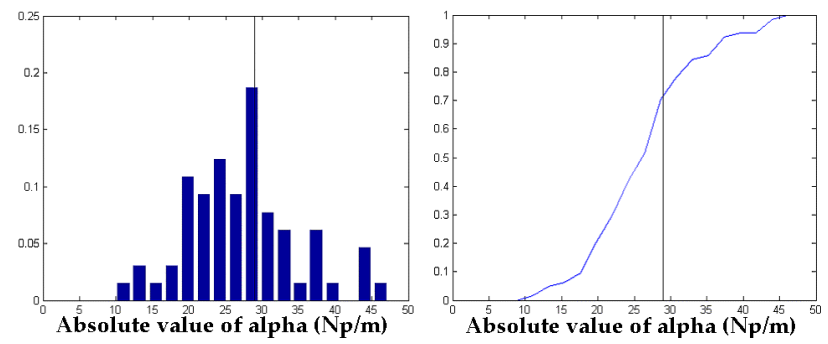

Figure 3. Absolute values of the maximum propagation “constant" $\alpha\left(\mathrm{Np} . \mathrm{m}^{-1}\right)$ at $900 \mathrm{MHz}$ for 64 phones

The calculation of the parameter $\alpha$ gives a quasi gaussian distribution of the values at $900 \mathrm{MHz}$, that allows evaluating precisely, from the distribution function, the $10 \%$ and $90 \%$ quantiles. At $1800 \mathrm{MHz}$, the distributions are more difficult to characterize in terms of statistical laws. At $900 \mathrm{MHz}$, determining this value is not difficult, the different values are very close, but at $1800 \mathrm{MHz}$ the measurement accuracy is poor, resulting in many different values for the propagation constant. The selected values of $\alpha$ representing the global decrease of the electric field in the phantom are equal to $23 \mathrm{~Np} / \mathrm{m}$ at $900 \mathrm{MHz}$ and $55 \mathrm{~Np} / \mathrm{m}$ at $1800 \mathrm{MHz}$. 


\section{Geometrical shape of the E-Field}

\section{E-field representation}

The characteristics of the propagation medium have an influence on the electromagnetic propagation, but also on the shape of the Efield. Statistically, the spatial distribution of the electric field, in more than $90 \%$ of the tested phones, is of ellipsoidal type. The Fig. 4 shows an example of E-field shapes for typical commercial phones in the plane close to the bottom of the phantom $(6 \mathrm{~mm}$ above it). The E-field data have been obtained with a meshing step of $7 \mathrm{~mm}$, in $70 \mathrm{~mm}$ size squares. The electric field shape can be almost of circular type, while the elliptic shape observed in the figures is more representative of the majority of the phones. Modeling the circular case, as a degenerate case of the ellipsoidal model, constitutes however an important step for the estimation of the parameters of the ellipsoidal model we have built to represent the distribution of the field in the phantom.

With the benefits of an important data base of SAR and E-field measurements for about hundred commercial phones, we assume that the distribution of the E-field in the phantom is much more simple than it could appear. The example given here under (Fig. 4) is characteristic of those met the most often in the data base.
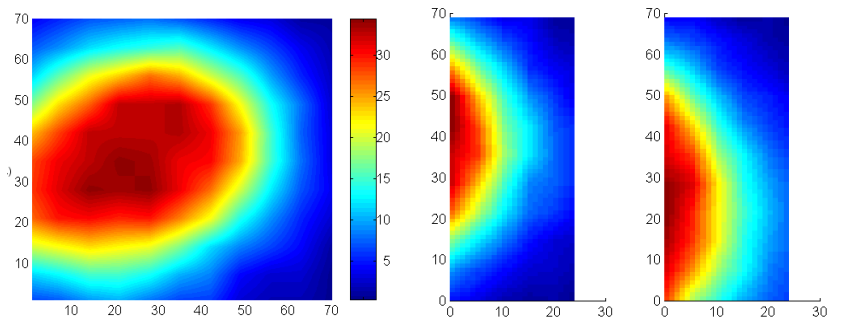

Figure 4. Depth decrease of the maximum normalized E-Field, for 64 phones at $1800 \mathrm{MHz}$.

\section{Degrees of freedom of the system}

The elliptic spatial distribution of the E-field and propagation as an exponential function of depth are characteristics relatively easy to model. These investigations let think that giving morphological and physical considerations on the E-field must allow introducing $a$ priori information in the SAR calculation [5]. The number of data points required in the standard way to compute the SAR should be decreased, by considering that a large number of those points depend from each other from electromagnetic or morphological laws. The electric field in the phantom can then be seen, in that way, as a relatively simple system: the E-field generated by a spherical wave expansion, as a first approximation. A first estimation of the degree of freedom $N$ of the distribution of the electric field in the phantom can be evaluated by the number of useful terms of its development in spherical modes [6]: $N=\lfloor k r\rfloor+10$, where $k=\frac{2 \pi f}{v_{\phi}}$ and where the square brackets indicate the largest integer smaller than or equal to $k r, \mathrm{r}$ is the radius of the domain of study i.e. the sphere of smallest radius that circumscribes the system, $v_{\Phi}$ the velocity of light, $f$ the frequency in the liquid. As an example, for $r=3.5 \mathrm{~cm}$ we obtain $\mathrm{N} \approx 14$ for $\mathrm{f}$ $=900 \mathrm{MHz}$, and $\mathrm{N} \approx 18$ for $\mathrm{f}=1800 \mathrm{MHz}$. We can then think that about 10 parameters of a mathematical model should be enough to reconstruct the E-field in the entire volume from a reduced number of data points.

\section{Modeling of the electric field}

\section{Ellipsoidal model}

In order to obtain a model close to the spatial distributions of the Efield observed, we developed an ellipsoidal model (3):

$$
\begin{aligned}
E\left(\overrightarrow{\mathbf{p}}_{e}, \overrightarrow{\mathbf{r}}\right) & =A_{0} \exp (-\alpha(1-\kappa) \\
& \left.\sqrt{\left(x_{m}-x_{e}\right)^{2}+\left(y_{m}-y_{e}\right)^{2}+\left(z_{m}-z_{e}\right)^{2}}\right)
\end{aligned}
$$

where $\rho(\overrightarrow{\mathbf{r}})=(1-\kappa) \sqrt{\left(x_{m}-x_{e}\right)^{2}+\left(y_{m}-y_{e}\right)^{2}+\left(z_{m}-z_{e}\right)^{2}}$,

$$
\kappa=\sqrt{\frac{1}{\frac{x^{\prime 2}}{a^{2}}+\frac{y^{\prime 2}}{b^{2}}+\frac{z^{\prime 2}}{c^{2}}}} \text { and } \mathbf{r}(x, y, z) \text { is the vector of positions }
$$

of the E-field measurement points in the phantom. The vector $\overrightarrow{\mathbf{r}}^{\prime}\left(x^{\prime}, y^{\prime}, z^{\prime}\right)=\mathbf{R}_{z}(\chi) \mathbf{R}_{y}(\beta) \mathbf{R}_{x}(\alpha) \overrightarrow{\mathbf{r}}^{\prime}(x, y, z)$ is obtained with the rotation matrices built from the Euler rotation angles $\alpha, \beta, \gamma$. $\mathbf{p}_{\mathrm{e}}\left(E_{0}, k, x_{\mathrm{s}}, y_{\mathrm{s}}, z_{\mathrm{s}}, a, b, c, \alpha, \beta, \gamma\right)$ is the vector of parameters, where $E_{0}$ represents the field amplitude, $k$ is similar to the propagation constant $\alpha, x_{\mathrm{s}}, y_{\mathrm{s}}$ and $z_{\mathrm{s}}$ are the coordinates of the centre of the ellipsoid, and $a, b$ and $c$ its semi-axes. $z_{\mathrm{s}}$ is constrained to be always negative. For physical reasons, the amplitude $E_{0}$ is positive, as well as $k . x_{\mathrm{s}}$ and $y_{\mathrm{s}}$ belong to $\Re$, and $a, b$ and $c$ are evidently positive. The Euler angles are taken into the range $[-\pi ; \pi]$.

\section{Hybrid unique propagation constant - ellipsoidal model}

Even if the preceding modelization technique ensure a very fast SAR measurement, we tried to go further by using the electromagnetic properties of the E-field developed in the previous sections. By using E-field values deduced from the propagation constant $\alpha$, instead of the corresponding real E-Field data points, the acquisition of data requires now only one plane of $4 \times 4$ E-field points. The Fig. 5 shows the standard 726 E-field points distribution and the ones used for the parametric reconstruction with only 21 points. We will adapt the 21 points distribution for the hybrid plane wave-ellipsoidal model, by using a plane of $16 \mathrm{E}$-field data points and calculating the 5 points in depth in the maximum field zone from plane wave propagation constant $\alpha$.
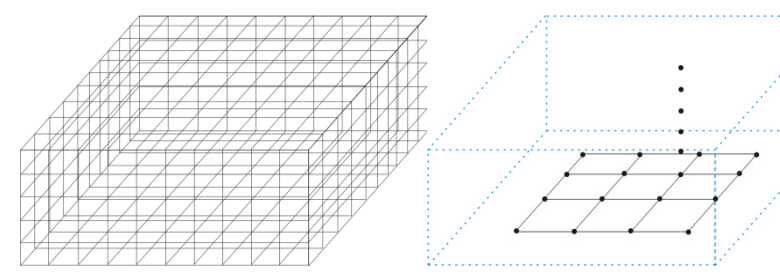

Figure 5. E-field points distribution in the phantom (standard method with 726 and reduced with 21 points)

\section{Parameters estimation}

The method of the parametric reconstruction of the E-field uses a mathematical model representing the field in order to reconstruct it in the phantom, from a little set of data points, with the desired meshing. The non-linear problem to solve carries a large number of unknowns, and has few given information. From the $n$ measurement points chosen, the parameters of the considered model are estimated in the sense of the least squares of the error between the model and the measurement points.

The 5 parameters of the spherical model are first estimated. The vector of parameters $\mathbf{p}_{\mathrm{s}}\left(E_{0}, k, x_{\mathrm{s}}, y_{\mathrm{s}}, z_{\mathrm{s}}\right)$ is then used as initialisation values for its common parameters with $\mathbf{p}_{\mathrm{e}}\left(E_{0}, k, x_{\mathrm{s}}, y_{\mathrm{s}}, z_{\mathrm{s}}, a, b, c, \alpha\right.$, $\beta, \gamma)$, for the estimation of the parameters of the ellipsoidal model. The other parameters of the ellipsoidal model $(a, b, c, \alpha, \beta, \gamma)$ are initialised with the median values of their respective variation ranges. With this technique, the convergence of the estimation of the ellipsoidal parameters is optimised, avoiding that the estimation lay in a local minimum too far from a good solution. In the estimation process, the parameters are normalized, in order to eliminate the scaling factors and then avoid the prevalence of some 
parameters. When the parameters have been determined, the calculation of every E-field point of the considered volume is immediate, as the standard procedure of computation of the maximum SAR integrated in $10 \mathrm{~g}$.

\section{Results on real phones data}

The two main reconstruction process have been tested on 128 measurements corresponding to 60 different mobile phones - 4 of them have retractable antenna (2 configurations) - for 2 frequencies. The following figures present the maximum averaged SAR on $1 \mathrm{~g}$ (U.S. standard) and $10 \mathrm{~g}$ (European standard), for observed and reconstructed data with 16 real data points plus 5 extrapolated points calculated with the plane wave propagation constant $\alpha$ (Fig. 6), 16 real data points plus 5 extrapolated points calculated with the mean propagation constant $\alpha$ (Fig. 7) and with 21 real data points (Fig. 8). Those results are compared to the "real" measurements obtained from 726 E-field data points. It must be noticed that the quality of the reconstruction is bound to the geometrical form of the real field, but also to its intensity. The method is less powerful if the observed field strength is near the probe's sensitivity. The accuracy of the method (the mean error is $10 \%$ for the 21 real data points) has to be compared with the complete error made with standard dosimetric facilities, which is commonly $30 \%$ to $40 \%$. The reproducibility between two measurements for the same phone and with the same assessment is about $5 \%$ to $10 \%$. The choice of an E-field propagation model based on real measurements is therefore better than the simplest description of the plane wave. This result confirm the previous observation of the relatively random decreases of the E-field as a function of depth. However, the approach using the plane wave propagation constant, modified by the use of lower propagation constants ensuring a maximized value for the calculated SAR, could nevertheless find an application in very fast measurements, for pre-compliance or systematic production tests for example.

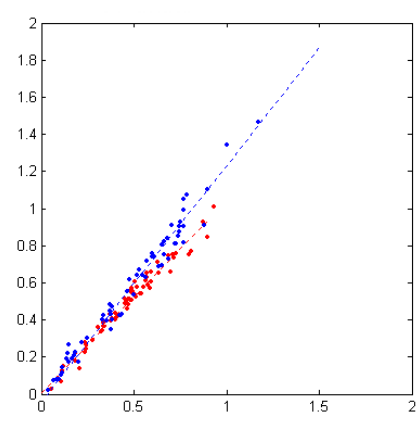

a)

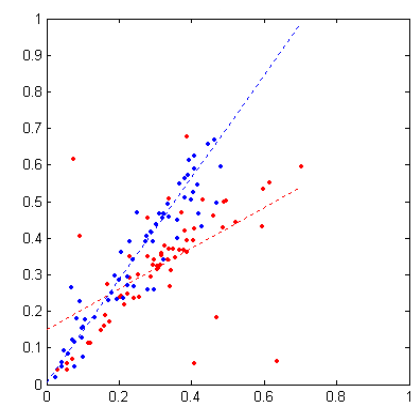

b)
Figure 6. Reconstructed SAR (W/kg) in function of the real SAR, integrated in $1 \mathrm{~g}$ (a) and in $10 \mathrm{~g}(\mathrm{~b})$, with $21 \mathrm{E}$-field points, the plane wave propagation constant and the ellipsoidal model

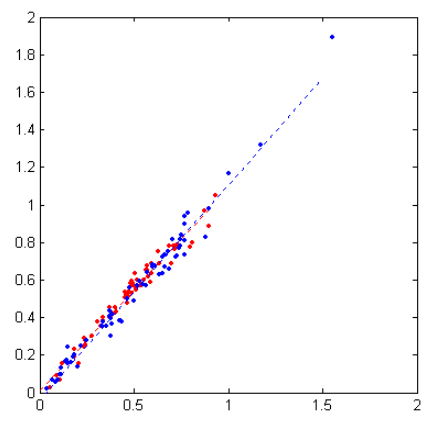

a)

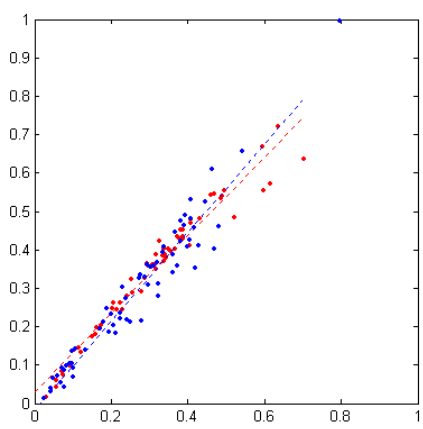

b)
Figure 7. Reconstructed $S A R(W / \mathrm{kg})$ in function of the real $S A R$, integrated in $1 \mathrm{~g}$ (a) and in $10 \mathrm{~g}(\mathrm{~b})$, with the mean propagation constant and the ellipsoidal model

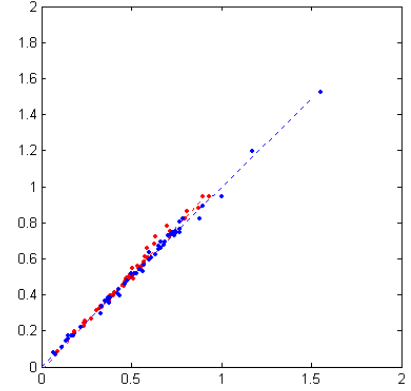

a)

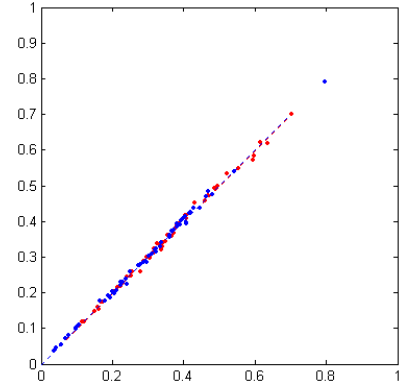

b)
Figure 8. Reconstructed SAR (W/kg) in function of the real SAR, integrated in $1 \mathrm{~g}$ (a) and in $10 \mathrm{~g}$ (b), with $36 \mathrm{E}$-field points in the phantom and the ellipsoidal model

\section{Conclusion}

This parametric approach employed in a novel application domain has yet proved itself with the robust estimation of other physical quantities [7]. The methods proposed in this paper, useful with standard dosimetric measurement systems, allows calculating the electric field and the SAR from a reduced number of observed data points. The local SAR is directly obtained from the knowledge of the model parameters. The maximum $10 \mathrm{~g}$ averaged SAR is then instantly obtained. The errors made on the $10 \mathrm{~g}$ averaged SAR, in the case of the full ellipsoidal propagation model, are much lower than the accuracy of the complete standard measurement itself. The measurement time for a given configuration is less than 1 minute (16 real points plus plane wave extrapolation), whereas the data processing time is neglectible. Furthermore, the hybrid ellipsoidalplane wave approach, used in conjunction with an array of E-field probes, should be able to provide quasi real time SAR measurement capabilities.

\section{References}

[1] Schmid, T., Egger, O., Kuster, N., Automated E-Field scanning system for dosimetric assessments, IEEE Transactions on Microwave Theory and Techniques, vol. $44, \mathrm{n}^{\circ} 1$, January 1996.

[2] prES 59005, Considerations for evaluation of human exposure to Electromagnetic Fields (EMFs) from Mobile Telecommunication Equipment (MTE) in the frequency range $30 \mathrm{MHz}-6 \mathrm{GHz}$, European standard, CENELEC, Bruxelles, December 1997.

[3] Manning, M., Massey, P., Rapid SAR Testing of Mobile Phone Prototype Using a Spherical Test Geometry, IEE Technical Seminar on Antenna Measurements and SAR (AMS 2002) May, 28-29 2002, Loughborough University, UK.

[4] Merckel, O., Fleury, G., Bolomey, J.-Ch., Rapid SAR Measurement via Parametric Modeling, 5th International Congress of the European BioElectromagnetics Association, p75-77, Helsinki, Finland, September 2001.

[5] Sandu, L., Oksman, J., Fleury, G., Information criteria for the choice of parametric functions for measurement, IEEE Transactions on Measurement, vol. 47, n4, pp. 920-924, 1998.

[6] Hansen, J.E., Spherical near-field antenna measurements, IEE Electromagnetic Waves Series 26., 1988.

[7] Fleury, G., Davoust, M.E., Remote Field Eddy Current Inspection for Groove Sizing Choice of a Direct Model Structure, Review of Progress in Quantitative Nondestructive Evaluation, American Institute of Physics, vol. 19A, n509, pp. 541-548, 1999. 